\title{
EFECTO GASTROPROTECTOR DE LOS FLAVONOIDES DEL EXTRACTO ETANÓLICO DE LAS PARTES AÉREAS DE Satureja sericea (goyal)
}

\author{
Gastroprotective effect of flavonoids in the ethanol extract of the aerial parts \\ of Satureja sericea "goyal"
}

\author{
Juvissan Aguedo A. ${ }^{1}$, Lidia Tinco O. ${ }^{1}$, Franco Rios H. ${ }^{1}$, Pablo Bonilla R. ${ }^{1}$, Jorge Arroyo A. ${ }^{2}$ \\ Instituto de Investigación en Ciencias Farmacéuticas y Recursos Naturales "Juan de Dios Guevara" \\ Facultad de Farmacia y Bioquímica. ${ }^{1}$ \\ Instituto de Investigaciones Clínicas y Laboratorio de Farmacología de la Facultad de Medicina. \\ Universidad Nacional Mayor de San Marcos. ${ }^{2}$
}

\begin{abstract}
RESUMEN
El "goyal" Satureja sericea, Lamiaceae, planta nativa de la comunidad de Mallas, provincia de Wari, departamento de Ancash, es usada como infusión de la parte aérea para aliviar afecciones de las vías digestivas debido a sus propiedades carminativas y antiinflamatorias. El Perú es un campo abierto para la investigación de su rica flora evaluada en 17144 especies (gimnospermas y angiospermas), 2458 géneros y 244 familias (Brako y Zarucchi, 1993), distribuida en la región costera, andina y amazónica. Para la presente investigación se colectó y estudió las partes aéreas de Satureja sericea (goyal), se secó bajo sombra, se pulverizó y se obtuvo el extracto por maceración hidroalcohólica. Se realizó marcha de solubilidad y marcha fitoquímica en la que se observó gran cantidad de compuestos fenólicos. Se comprobó la presencia de por lo menos cuatro compuestos fenólicos tipo flavonoides, mediante reacciones de coloración y precipitación, fueron aislados mediante cromatografía en capa fina a escala preparativa y se elucidó su estructura mediante espectroscopia UV visible en etanol y con reactivos de desplazamiento. En el extracto hidroalcohólico se determinó el efecto gastroprotector mediante el método descrito por Zhang et al. y Liu et al. en ratas albinas Holztman. Previo ayuno sólido de 24 horas, se seleccionaron cinco grupos y se administró por canulación orogástrica. Se obtuvo a dosis de $1000 \mathrm{mg}$ del extracto hidroalcohólico/Kg de peso una gastroprotección de un $95 \%$ con respecto al estándar de comparación.
\end{abstract}

Palabras clave: Satureja sericea, efecto gastroprotector, compuestos fenólicos, flavonoides.

\section{SUMMARY}

The "goyal" Satureja sericea, Lamiaceae, native plant of the department of Ancash province of Wari, community Mallas, is used as an infusion the aerial parts to relieve the carrier of ailments digestive tract because of its anti-inflammatory properties and carminativa. Peru is an open field for investigation of its rich flora assessed on 17,144 species (angiosperms and gymnosperms), 2458 genera and 244 families (Brako and Zarucchi, 1993), distributed in the coast region, Andean and Amazon. For this investigation is collected and studied the aerial parts of Satureja sericea (goyal), is dried under shade, is pulverized and the extract was obtained by macerating hydroalcoholic. Was conducted progress solubility and phyto march in which he observed large amounts of phenolic compounds. It was found the presence of at least four kinds phenolic flavonoids, through reactions of coloration and precipitation were isolated by thin layer chromatography and preparative scale to be elucidated its structure by UV-visible spectroscopy in ethanol and reagents displacement. In the excerpt hydroalcoholic gastroprotective effect was determined by the method described by Zhang et al. and Liu et al. Holztman in albino rats. Prior fasting for 24 hours solid, five groups were selected and administered by cannulation. We obtained a dose of $1000 \mathrm{mg}$ of hydroalcoholic extract $/ \mathrm{kg}$ of a gastroprotection $95 \%$ compared with the standard.

Keywords: Satureja sericea, gastroprotective effect, phenolic compounds, flavonoids.

A quien debe dirigirse la correspondencia. E-mail: pabloenriquebr@yahoo.com 


\section{INTRODUCCIÓN}

Satureja sericea, comúnmente conocida como "goyal", de la familia Lamiaceae, es una planta nativa del departamento de Ancash, provincia de Wari, específicamente crece en la comunidad de Mallas, y que según los datos proporcionados por la etnobotánica, la medicina popular y antropológica, indican sus usos como infusiones de la parte aérea para aliviar afecciones de las vías digestivas, por lo que es utilizada por los pobladores de la mencionada comunidad debido a sus propiedades carminativas y antiinflamatorias. El empleo de plantas con fines curativos es una práctica que se ha utilizado desde tiempos inmemoriales. Los principios activos en las plantas, se hallan siempre biológicamente equilibrados por la presencia de sustancias complementarias, que van a potenciarse entre sí, de forma que en general no se acumulan en el organismo, y sus efectos indeseables están limitados (1).

Actualmente las enfermedades gástricas y duodenales constituyen un serio problema de salud en nuestro país, debido a que su incidencia ha aumentado en las últimas décadas por los hábitos de comidas rápidas con exceso de grasas trans, condimentos y colorantes sintéticos y el abuso de consumo de café, té, gaseosas, alcohol, además de diferentes medicamentos que ocasionan las úlceras medicamentosas. Muchos enfermos no presentan síntomas, hay un 20 a $30 \%$ de pacientes que pueden hasta sufrir una perforación o una hemorragia sin manifestación alguna. Esta enfermedad afecta en su mayoría a personas que oscilan entre 35 y 48 años de edad, aunque también suele presentarse en niños, adolescentes y ancianos. Cuando se presenta perforación o hemorragia súbita, puede comprometer la vida del paciente si no se trata a tiempo. Los pacientes con ulcera duodenal se quejan de dolor y una sensación corresiva en la zona media del epigastrio o en el dorso. Estas personas secretan un exceso de jugo gástrico que contiene más ácido y pepsina que lo normal (2). El Perú es un campo abierto para la investigación de su rica flora evaluada en 17144 especies (gimnospermas y angiospermas), 2458 géneros y 244 familias (Brako y Zarucchi, 1993) (10), distribuida en la región costera, andina y amazónica (3).

En una búsqueda constante de los investigadores de encontrar principios activos para el tratamiento de afecciones gástricas, al presente, se hace necesario buscar metabolitos secundarios o compuestos químicos procedentes de productos naturales con actividad antiinflamatoria y para desórdenes digestivos Basten, 1981(4); Gupta, 1995 (5), como es el caso de los flavonoides que son compuestos fenólicos distribuidos ampliamente en la naturaleza y han demostrado tener una gran actividad biológica, principalmente como antioxidante y antiinflamatorio (Lock de Ugaz, 1994)(6), (Villar del Fresno, 1999)(7), (Kuklinski, 2000)(8) (Bruneton, 2001)(9). El presente trabajo de investigación pretende demostrar la acción gastroprotectora de los metabolitos secundarios presentes en el extracto hidroalcohólico de las partes aéreas de Satureja sericea (goyal).

\section{GENERALIDADES}

\section{Descripción botánica}

Satureja sericea es un arbusto de $50-150 \mathrm{~cm}$ de alto. Tallo cuadrangular, ligeramente estriado, pubescente con pequeños pelos ramificados. Hojas opuestas, simples, pecíolo 3-8 mm de largo, limbo ovado y ovado lanceolado 15-20 X 8-10 $\mathrm{mm}$, penninervado, envés seríceo argentado, haz verdoso, ápice apiculado. Inflorescencias cimas axilares y verticilastros, algunas flores solitarias. Flor heteroclamídea, hermafrodita, zigomorfa; cáliz bilabiado 7-10 X 3-5 mm, 5 lóbulos agudos, 3 en el labio superior y 2 en el inferior; corola roja o anaranjada 25-27 x 5-7 mm, lóbulos obtusos, 2 en el labio superior y 3 en el inferior; estambre 4, didínamos insertos en la base del tubo de la corola; ovario bicarpelar, bilocular con 2 rudimentos seminales en cada lóculo, por la aparición de falso tabique se hace tetralocular. Fruto tetraclusa. Su Hábitat es del tipo de ladera rocosa (10).

\section{Características generales del área de recolección}

En la comunidad de Mallas ubicada en la provincia y distrito de Huari departamento de Ancash, comprende $131 \mathrm{Km} 2$, latitudinalmente se ubica entre los $9^{\circ} 23^{\prime} 30^{\circ}$ L.S. y $77^{\circ} 11^{\prime} 20^{\circ}$ L.O. El área situada por encima de los $3550 \mathrm{msnm}$, hacia el lado oriental de la Cordillera Blanca, esta circunscrita en el perímetro del Parque Nacional del Huascarán. 


\section{Aspecto climático}

Como en la mayoría de valles andinos el clima es templado, en los niveles altos, por encima de los $3600 \mathrm{msnm}$, como en la puna y en las cumbres el frió es glacial.

No se reportan estudios de la Satureja Saricea por este motivo, el presente trabajo de investigación esta orientado a comprobar las propiedades medicinales que se le atribuyen, mediante el uso de diversas técnicas, que nos permitan identificar los distintos metabolitos secundarios y relacionarlos con la actividad farmacológica, se hizo la investigación en los laboratorios del Instituto de Investigación en Ciencias Farmacéuticas y Recursos Naturales "Juan de Dios Guevara" de la Facultad de Farmacia y Bioquímica, y en el laboratorio del Instituto de Investigaciones Clínicas y Farmacología Experimental de la Facultad de Medicina de la Universidad Nacional Mayor de San Marcos de Lima. Los objetivos trazados fueron determinar el efecto gastroprotector del extracto hidroalcohólico de las partes aéreas de la Satureja serícea y obtener los metabolitos secundarios tipo flavonoides de dicho extracto.

\section{MATERIALES Y MÉTODOS}

Material básico de vidrio

Material biológico

- Ratas albinas holtzmann machos de $210 \pm 20 \mathrm{~g}$

- Extracto hidroalcohólico de Satureja sericea (goyal)

Equipos e instrumental

- Equipo de disección completo (pinzas, tijeras, etc.)

- Espectrofotómetro UV- visible. Hewlett Packard.

- Equipo de Cromatografía de capa fina. Lámpara de Luz ultravioleta $265 \mathrm{~nm}$ y $354 \mathrm{~nm}$

Material Farmacológico

- Extracto hidroalcohólico de Satureja sericea (goyal)

- Suero fisiológico

- Buffer fosfato pH 7

Reactivos

- Solventes: éter de petróleo, diclorometano $\mathrm{QP}$, acetato de etilo QP, butanol QP, etanol QP, metanol QP , agua destilada.
- Reactivos de Desplazamiento: metóxido de sodio, tricloruro de aluminio, ácido clorhídrico, acetato de sodio.

\section{METODOLOGÍA}

La muestra de "goyal" se colectó en la comunidad de Mallas, Huari, Ancash. Se seleccionaron las partes aéreas (hojas y ramas) se secaron al medio ambiente bajo sombra, luego fueron molidas en un molino eléctrico a cuchillas y almacenadas en frascos de vidrio de color ámbar para su posterior tratamiento $(6,11)$.

\section{Obtención del extracto}

La extracción se realizó al macerar $500 \mathrm{~g}$ de polvo seco de la parte aérea de goyal, en 3 it de etanol al $70 \%$, durante 8 días con constante agitación, luego se filtró y concentró a sequedad en una estufa de aire circulante a $40^{\circ} \mathrm{C}(6,11)$.

\section{Marcha de solubilidad}

Con el extracto seco hidroalcohólico, se hizo una marcha de solubilidad con solventes de polaridad creciente: éter de petróleo, diclorometano, acetato de etilo, butanol, etanol, metanol, agua destilada $(6,11)$.

\section{Estudio fitoquímico}

Se realizó la marcha fitoquímica del extracto hidroalcohólico con los reactivos generales de coloración y precipitación. Luego se realizó la cromatografía en capa fina analítica para la detección e identificación de los metabolitos secundarios presentes y cromatografía en capa fina a escala preparativa para aislar los componentes mayoritarios, utilizando como fase fija silicagel F254. Se preparó dos sistemas de fases móviles: diclorometano: metanol $(3: 1)$ y diclorometano: metanol (6:1) y se obtuvieron varias fracciones, de las cuales, fueron aisladas cuatro con etanol de $96^{\circ}(6,11)$. Se elucidó la estructura química de las cuatro fracciones aisladas mediante espectrofotometría ultravioleta con etanol como solvente, y con reactivos de desplazamiento, metóxido de sodio, tricloruro de aluminio, ácido clorhídrico, acetato de sodio, para diferenciar las posiciones de los grupos presentes en los núcleos químicos básicos de los metabolitos secundarios presentes $(9,12)$. 


\section{Determinación del efecto gastroprotector}

En el extracto hidroalcohólico se determinó el efecto gastroprotector mediante el método descrito por Zhang et al 1995 (13) y Liu et al. 2002 (14) en ratas albinas holtzmann. Se utilizaron 30 ratas machos de $210 \pm 20 \mathrm{~g}$ procedentes del Bioterio del Instituto Nacional de Salud. Los animales previamente fueron aclimatados al ambiente de experimentación durante 5 días, se mantuvieron en un ambiente a temperatura de $21^{\circ} \mathrm{C}$ con dieta y agua a libertad. Veinticuatro horas antes del ensayo, se les sometió a ayuno, recibiendo agua a libertad. Se dividieron en cinco grupos de seis, según el siguiente diseño experimental:

1. Grupo control normal: solución suero fisiológico (SSF) $2 \mathrm{~mL} / \mathrm{kg}+\mathrm{SSF} 2 \mathrm{~mL} / \mathrm{kg}$

2. Grupo control úlcera: SSF $2 \mathrm{~mL} / \mathrm{kg}+$ etanol (E) $1 \mathrm{~mL}$ por rata.

3. Grupo control úlcera más dosis 1: Extracto hidroalcohólico $25 \mathrm{mg} / \mathrm{Kg}$ + E $1 \mathrm{~mL}$ por rata.

4. Grupo control úlcera más dosis 2: Extracto hidroalcohólico $250 \mathrm{mg} / \mathrm{Kg}+\mathrm{E} 1 \mathrm{~mL}$ por rata.

5. Grupo control úlcera más dosis 3: Extracto hidroalcohólico $1000 \mathrm{mg} / \mathrm{Kg}+\mathrm{E} 1 \mathrm{~mL}$ por rata.

El procedimiento fue el siguiente, mediante sonda metálica de acero inoxidable, en primer lugar se administraron por vía oral el suero fisiológico y el extracto en las diferentes dosificaciones antes indicadas. Luego de 60 minutos, se administró el etanol a todos los grupos, excepto al grupo normal $(13,14)$. Luego de dos horas, los animales fueron sacrificados, por administración de pentobarbital $100 \mathrm{mg} / \mathrm{kg}$ vía intraperitoneal; mediante laparotomía se extrajeron los estómagos, los que se abrieron por la curvatura mayor, lavándose suavemente con suero fisiológico con la finalidad de retirar los detritus; observándose la presencia de lesiones, considerando: hiperemia, hemorragia, y úlceras; finalmente los estómagos fueron conservados en una solución de formol tamponado ( $100 \mathrm{~mL}$ de formol $40 \%+$ sodio fosfato monobásico monohidratado $(4 \mathrm{~g})+$ sodio fosfato dibásico anhidro $(6.5 \mathrm{~g})+$ agua destilada csp un litro). Se consideró la escala de Ninguno $=0$; leve $=1 ;$ moderado $=2 ;$ severo $=3$.

\section{RESULTADOS}

Tabla 1. Marcha de solubilidad del extracto hidroalcohólico de Satureja sericea (goyal)

\begin{tabular}{|c|c|}
\hline Solvente & Resultado \\
\hline Éter de petróleo & - \\
\hline Diclorometano & - \\
\hline Acetato de etilo & - \\
\hline $\mathrm{N}$ - butanol & + \\
\hline Etanol & + \\
\hline Metanol & ++ \\
\hline Agua & ++ \\
\hline
\end{tabular}

(-) Insoluble, (+) Ligeramente Soluble, $(++)$ Soluble

Tabla 2.

Marcha fitoquímica del extracto hidroalcohólico de Satureja sericea (goyal)

\begin{tabular}{|l|c|c|}
\hline \multicolumn{1}{|c|}{ Reactivo } & Resultado & Presencia de: \\
\hline Gelatina & + & Taninos \\
\hline Ninhidrina & + & Aminoácidos libres \\
\hline Tricloruro férrico & +++ & Compuestos fenólicos \\
\hline NaOH 5\% (Bortranger) & + & Quinonas \\
\hline Mayer & + & Alcaloides \\
\hline Drangendorff & + & Alcaloides \\
\hline Naftol $+\mathrm{H}_{2} \mathrm{SO}_{4 \mathrm{c}}($ Molisch) & + & Glicósidos \\
\hline $\begin{array}{l}\text { Mg metálico }+\mathrm{HClc} \\
\text { Shinoda) }\end{array}$ & +++ & Flavonoides \\
\hline Lieberman & & \\
\hline
\end{tabular}

$(+++)$ abuindante, $(++)$ regular, $(+)$ poco 
Tabla 3.

Resultados de los espectros UV-visible de cuatro compuestos aislados del extracto hidroalcohólico de Satureja sericea (goyal) (12)

M1:

$\begin{array}{lllll}\lambda_{\text {máx }}^{\mathrm{EtOH}} & : 286,336 \mathrm{~nm} ; \lambda_{\text {máx }}^{\mathrm{Metax} N a}: 278,374 \mathrm{~nm} ; \lambda_{\text {máx }}^{\mathrm{EtOH}+\mathrm{AlCl}_{3}} & : 282,390 \mathrm{~nm} ; \\ \lambda_{\text {máx }}^{\mathrm{EtOH}+\mathrm{AlCl}_{3} \mathrm{HCl}}: 290,336 \mathrm{~nm} ; \lambda_{\text {máx }}^{\mathrm{EtOH}+\mathrm{Actato}} & : 284,330,400 \mathrm{~nm} & \\ \mathrm{M} 2: & & & \\ \lambda_{\text {máx }}^{\mathrm{EtOH}} & : 278,330 \mathrm{~nm} ; \lambda_{\text {máx }}^{\mathrm{Metox} \mathrm{Na}}: 274,364 \mathrm{~nm} ; \lambda_{\text {máx }}^{\mathrm{EtOH}+\mathrm{AlCl}_{3}} & : 278,330 \mathrm{~nm} \\ \lambda_{\text {máx }}^{\mathrm{EtOH}+\mathrm{AlCl}_{3} \mathrm{HCl}}: 278,330 \mathrm{~nm} ; \lambda_{\text {máx }}^{\mathrm{EOHH}+\mathrm{Actato}} & : 278,330 \mathrm{~nm} & \end{array}$

M3:

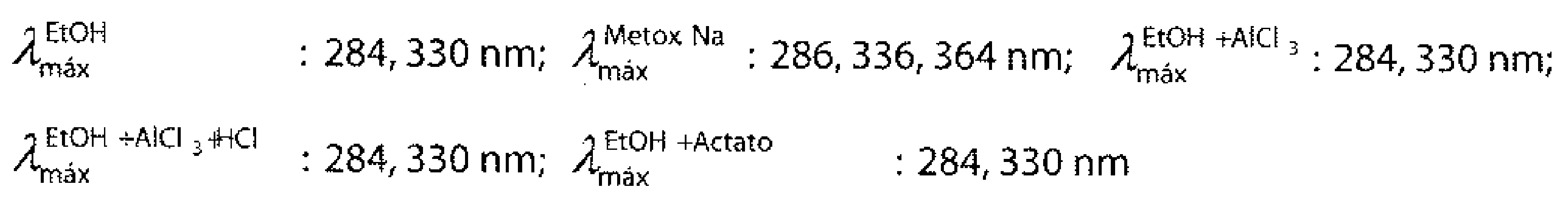

M4:

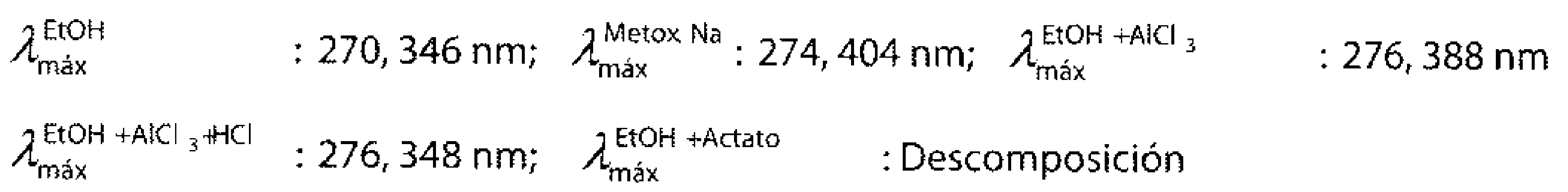

Tabla 4.

Efecto gastroprotector del extracto hidroalcohólico de Satureja sericea (goyal), en vista macroscópica y su puntuación según escala.

\begin{tabular}{|l|l|l|l|}
\hline Grupo & Dosis & Protección & Características \\
\hline Control normal & $\begin{array}{l}\text { Suero fisiológico } \\
(0,5 \mathrm{~mL})\end{array}$ & --- & $\begin{array}{l}\text { Mucosa de estructura conservada. } \\
\text { Hiperemia }=0 \\
\text { Hemorragia }=0 \\
\text { Ulceras }=0\end{array}$ \\
\hline Control álcera & Etanol $(0,2 \mathrm{~mL})$ & -- & $\begin{array}{l}\text { Zonas hemorrágicas, congestionadas e } \\
\text { hiperémicas. Vasos coagulados. Sangrado e } \\
\text { infección aguda. Erosión de capilares y vasos. } \\
\text { Hiperemia }=3 \\
\text { Hemorragia }=3 \\
\text { Ulceras }=3\end{array}$ \\
\hline $\begin{array}{l}\text { Control úlcera } \\
\text { más dosis 1 }\end{array}$ & $25 \mathrm{mg} / \mathrm{Kg}$ & $30-40 \%$ & $\begin{array}{l}\text { Zonas hemorrágicas y congestivas. } \\
\text { Hiperemia }=2 \\
\text { Hemorragia }=2 \\
\text { Ulceras =3 }\end{array}$ \\
\hline $\begin{array}{l}\text { Control úlcera } \\
\text { más dosis } 2\end{array}$ & $250 \mathrm{mg} / \mathrm{Kg}$ & $40-50 \%$ & $\begin{array}{l}\text { Algunas zonas hiperémicas y de congestión. } \\
\text { Hiperemia }=2 \\
\text { Hemorragia }=2 \\
\text { Ulceras }=2\end{array}$ \\
\hline $\begin{array}{l}\text { Control úlcera } \\
\text { más dosis } 3\end{array}$ & $1000 \mathrm{mg} / \mathrm{Kg}$ & $\begin{array}{l}\text { Se aprecia capilares, mucosa y células } \\
\text { normales. No hay evidencia de sangrado. } \\
\text { Hiperemia }=1 \\
\text { Hemorragia }=1 \\
\text { Ulceras = 1 }\end{array}$ \\
\hline
\end{tabular}

Escala de puntuación: Se consideró la escala de Ninguno $=0 ;$ leve $=1 ;$ moderado $=2$; severo $=3$. 
Figura 1.

Efecto gastroprotector según escala de Liker, de las lesiones gástricas inducidas por etanol en ratas machos

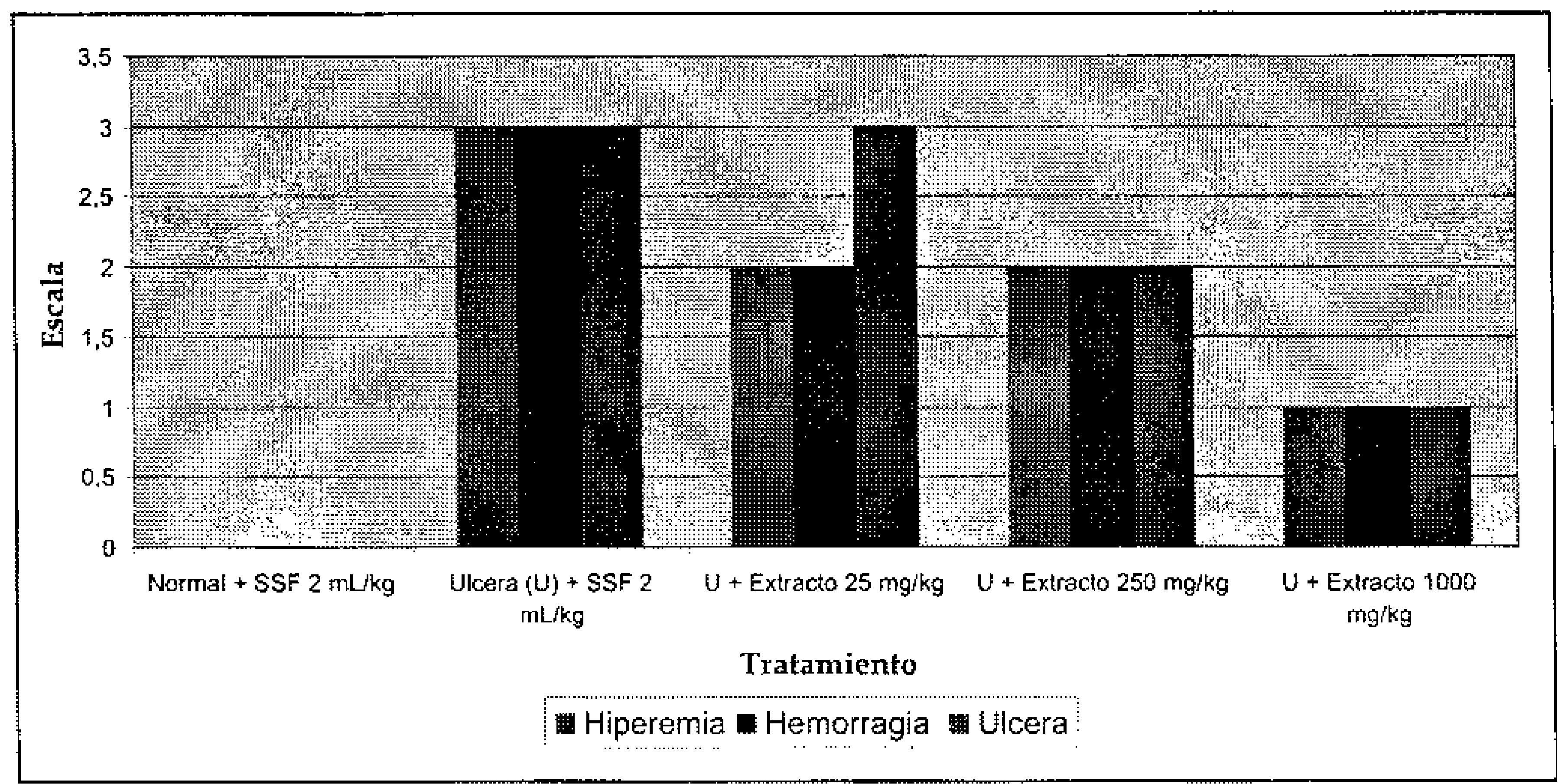

Tabla 5.

Efecto gastroprotector del extracto hidroalcohólico de Satureja sericea (goyal), en vista Microscópica

\begin{tabular}{|l|c|c|}
\hline \multicolumn{1}{|c|}{ Grupo } & Dosis & $\begin{array}{c}\text { Protección } \\
(\%)\end{array}$ \\
\hline Control normal & $\begin{array}{c}\text { Suero fisiológico } \\
(0,5 \mathrm{~mL})\end{array}$ & --- \\
\hline Control úlcera & \begin{tabular}{c} 
Etanol $(0,2 \mathrm{~mL})$ \\
\hline $\begin{array}{l}\text { Control úlcera } \\
\text { más dosis 1 }\end{array}$
\end{tabular} & --- \\
\hline $\begin{array}{l}\text { Control úlcera } \\
\text { más dosis 2 }\end{array}$ & $25 \mathrm{mg} / \mathrm{Kg}$ & 35 \\
\hline $\begin{array}{l}\text { Control úlcera } \\
\text { más dosis 3 }\end{array}$ & $1000 \mathrm{mg} / \mathrm{Kg}$ & 45 \\
\hline
\end{tabular}


Figura 2.

Lesión gástrica inducida por etanol $96^{\circ}$ en ratas machos

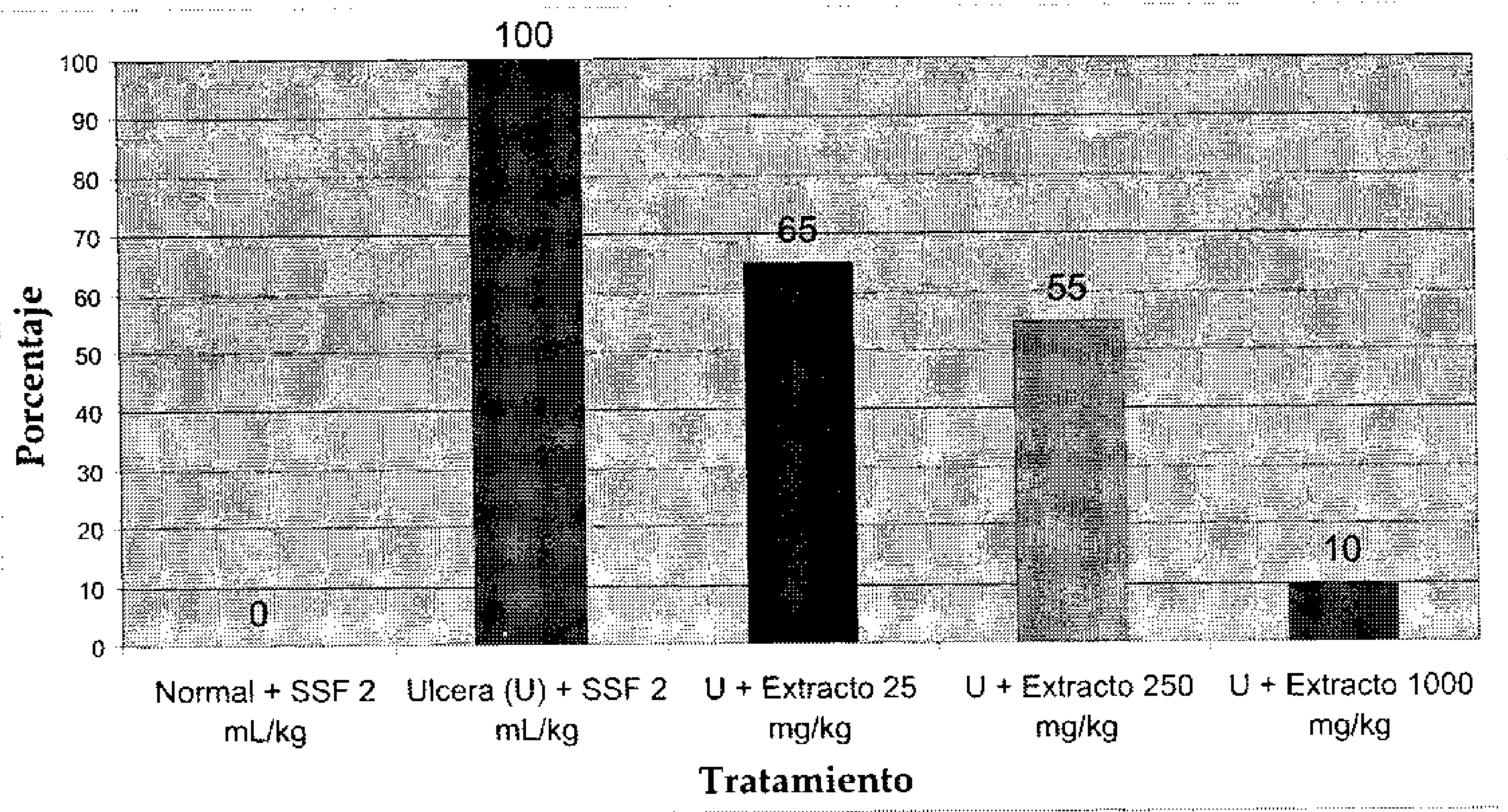

\section{DISCUSION}

La marcha de solubilidad permite establecer que el extracto hidroalcohólico de Satureja sericea contiene mayormente metabolitos secundarios solubles en butanol, etanol, metanol y agua; lo que indicaría que los componentes son de alta polaridad, siendo insolubles en el resto de solventes (tabla 1) $(6,11)$.

\section{Estudio fitoquímico}

El extracto hidroalcohólico de Satureja sericea muestra abundantes compuestos fenólicos tipo flavonoides y presencia de otros metabolitos secundarios tales como alcaloides, taninos, etc. Como se puede observar en la tabla $2(6,11)$.

\section{Análisis cromatográfico}

La cromatografía en capa fina a escala preparativa realizada en los extractos de las partes aéreas de la Satureja sericea, da como resultado una mejor separación con el sistema de solventes diclorometano: metanol $(6: 1)$, aislándose 4 fracciones.

\section{Elucidación de estructuras}

Mediante el ensayo de solubilidad y la marcha fitoquímica se encontró abundante cantidad de compuestos fenólicos en el extracto hidroalcohólico de las partes aéreas de Satureja sericea "goyal" (tabla 1,2). Mediante ensayos cromatográficos se detectaron e identificaron metabolitos secundarios tipo flavonoides, los que fueron confirmados por reacciones de coloración y precipitación. Los metabolitos secundarios denominados M1, M2, M3 y M4 fueron aislados por cromatografía en capa fina a escala preparativa, separados en etanol $96^{\circ}$ y luego leídos al espectrofotómetro UV-Visible en etanol y con varios reactivos de desplazamiento químico, según Mabry et al (12). Al interpretar los espectros obtenidos se observó que M1, M2, M3 y M4 correspondian a compuestos fenólicos tipo flavonoides y específicamente a derivados de flavonas, se propone las siguientes estructuras por la correlación espectral con los reactivos de desplazamiento (tabla 3) y por comparación con la información proporcionada por Mabry et al (12): 


$$
\begin{array}{ll}
M 1: & : 286,336 \mathrm{~nm} \\
\lambda_{\text {máx }}^{\mathrm{EtOH}} & : 278,374 \mathrm{~nm} \\
\lambda_{\text {máx }}^{\text {Metox Na }} & : 282,390 \mathrm{~nm} \\
\lambda_{\text {máx }}^{\mathrm{EtOH}+\mathrm{AlCl}_{3}} & : 290,336 \mathrm{~nm} \\
\lambda_{\text {máx }}^{\mathrm{EOH}+\mathrm{AlCl}_{3}+\mathrm{HCl}} & : 284,330,400 \mathrm{~nm} \\
\lambda_{\text {máx }}^{\text {EOH +Actato }} &
\end{array}
$$

Según el espectro en etanol el esqueleto básico que corresponde a esta fracción, es de una flavona (Fig.3), pues al tratarlo con metóxido de sodio hay un efecto hipsocrómico de $8 \mathrm{~nm}$ y un efecto batocrómico de $38 \mathrm{~nm}$ lo que nos indicaría que el compuesto tiene hidroxilos libres, principalmente en la posición $4^{\prime}$ y 7 , al hacer reaccionar con tricloruro de aluminio se observa un efecto batocrómico de $54 \mathrm{~nm}$, que luego al tratarlos con $\mathrm{HCl}$

$$
\begin{array}{ll}
\mathrm{M} 2: & : 278,330 \mathrm{~nm} \\
\lambda_{\text {máx }}^{\mathrm{EOH}} & : 274,364 \mathrm{~nm} \\
\lambda_{\text {máx }}^{\mathrm{Metox} \mathrm{Na}} & : 278,330 \mathrm{~nm} \\
\lambda_{\text {máx }}^{\mathrm{EOH}+\mathrm{AlCl}_{3}} & : 278,330 \mathrm{~nm} \\
\lambda_{\text {máx }}^{\mathrm{EOH}+\mathrm{AlCl}_{3}+\mathrm{HCl}} & : 278,330 \mathrm{~nm} \\
\lambda_{\text {máx }}^{\mathrm{EtOH}+\mathrm{Actato}^{2}} &
\end{array}
$$

Según el espectro en etanol el esqueleto básico que corresponde a esta fracción es de una flavo. na (Fig.4), pues al tratarlo con metóxido de sodio hay un efecto bactocromico de $34 \mathrm{~nm}$ lo que nos indicaría que el compuesto tiene hidroxilos libres, principalmente en la posición 7 , al hacer reaccionar con tricloruro de aluminio no se ob-<smiles>COc1c(O)c(OC)c2oc(-c3ccc(O)c(O)c3)cc(=O)c2c1O</smiles>

Figura 3.

M1. 3',4'5,7-tetrahidroxi- 6,8- di metoxi flavona

regresa al valor de la segunda señal esto nos indica presencia de hidroxilos libres en las posiciones $3^{\prime} y 4^{\prime}$; en la posicion 5 existe un $\mathrm{OH}$ libre que forma un complejo con el tricloruro de aluminio que al ser tratados con el ácido no se rompe por lo que no regresa a su valor original. Al tratarlo con acetato de sodio no se observa descomposición.<smiles>COc1ccc(-c2cc(=O)c3c(OC)c(OC)c(O)cc3o2)cc1</smiles>

Figura 4.

M2. 7- hidroxi- 3, $, 5,6$ - tri metoxi flavona

servan efectos batocrómicos y luego al tratarlos con $\mathrm{HCl}$ se mantienen las señales, esto nos indica que en posición 5 no existe $\mathrm{OH}$ libre para que forme un complejo con el tricloruro de aluminio. Al tratarlo con acetato de sodio no se observa descomposición.<smiles>COc1ccc(-c2cc(=O)c3c(OC)c(OC)c(O)c(OC)c3o2)cc1</smiles>

Figura 5.

M3. 7-hidroxi- 3',5,6,8-tetra metoxi flavona 
Según el espectro en etanol el esqueleto básico que corresponde a esta fracción es de una flavona (Fig. 5), pues al tratarlo con metóxido de sodio hay un efecto batocrómico de $34 \mathrm{~nm}$ lo que nos indicaría que el compuesto tiene hidroxilo libre en posición 7, al hacer reaccionar con tricloruro de aluminio no se observa efecto batocrómico, y luego al tratarlo con $\mathrm{HCl}$ tampoco hay variación, esto nos indica ausencia de hidroxilos libres en las posiciones 5 y 6 , al tratarlo con acetato de sodio se observa descomposición en el espectro lo que indicaría presencia de $\mathrm{OH}$ libre en 7 , confirma nuestra presunción.

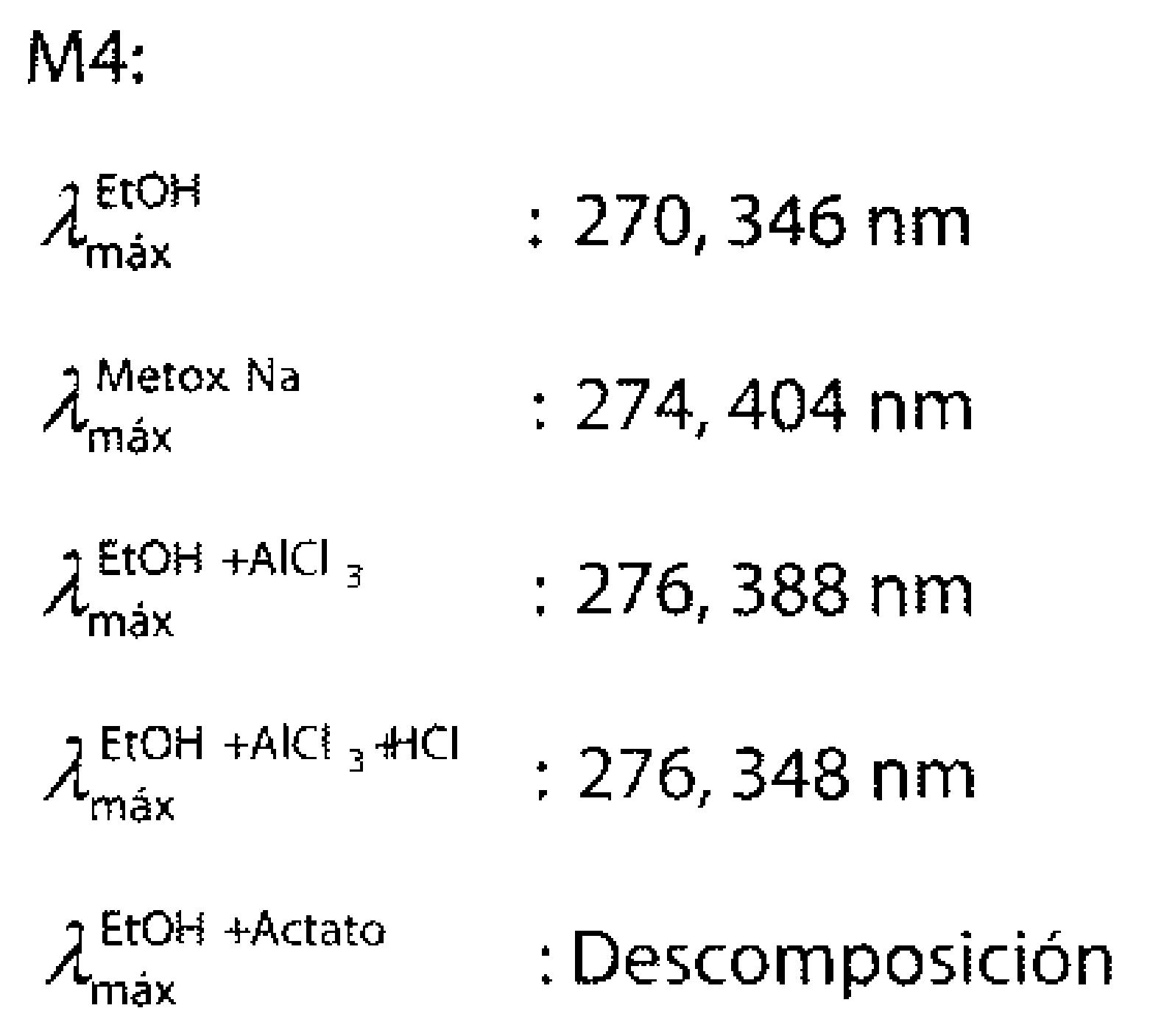

Según el espectro en etanol el esqueleto básico que corresponde a esta fracción es de una flavona (Fig.6), pues al tratarlo con metóxido de sodio hay un efecto batocrómico de $58 \mathrm{~nm}$ lo que nos indicaría que el compuesto tiene hidroxilos libres, principalmente en la posición $4^{\prime}$ y 7 , al hacer reaccionar con tricloruro de aluminio se observa un efecto batocrómico de $42 \mathrm{~nm}$, que luego al tratarlo con $\mathrm{HCl}$ regresa al valor de la segunda señal esto nos indica presencia de hidroxilos libres en las posiciones $3^{\prime}$ y $4^{\prime}$; en la posición 5 existe un $\mathrm{OH}$ libre que forma un complejo con el tricloru. ro de aluminio, por lo que se observó un ligero efecto batocrómico de $6 \mathrm{~nm}$, que al ser tratado con el ácido no se rompe por lo que no regresa a su valor original. Al tratarlo con acetato de sodio no se observa descomposición.

\section{Actividad gastroprotectora}

La moderna terapia para controlar la úlcera péptica es inhibir la secreción ácida gástrica, promover la gastroprotección, bloquear la apoptosis y estimular la proliferación de las células epiteliales para una efectiva cicatrización de la úlcera. (Bandhopadhyay et al., 2002) (18).

El etanol administrado a ratas posiblemente ha mostrado un incremento en el plasma de concentraciones de gastrina, esta hormona gástrica aumenta en la mucosa gástrica la actividad de la $\mathrm{H}+\mathrm{K}+\mathrm{ATPasa}$. La $\mathrm{H}+\mathrm{K}+\mathrm{ATPasa}$ es una enzima dimérica responsible para la secreción de ácido<smiles>COc1c(O)cc(O)c2c(=O)cc(-c3ccc(O)c(O)c3)oc12</smiles>

Figura 6.

M4. $3^{\prime}, 4^{\prime}, 5,7$ - tetrahidroxi- 8 - metoxi flavona

a nivel de las células parietales. $\mathrm{La} \mathrm{H}+\mathrm{K}+\mathrm{ATPasa}$ es selectivamente bloqueada por inhibidores de la bomba como el lanzoprazole siendo usado en el tratamiento de la úlcera péptica, (Nagaya et al., 1990) (19). La activación de la vía del AMPc estimula la $\mathrm{H}+\mathrm{K}+\mathrm{ATPasa}$ de las células parietales, una alta capacidad de la bomba de protones, con su inserción en l membrana apical conduce a la formación de un canalículo secretor (Brunton, 1996) (20). Recientemente los fármacos que reducen la secreción ácida e inhiben a la $\mathrm{H}+\mathrm{K}+\mathrm{ATPasa}$ son preferidos para uso clínico (David Perlin, 1998) (21). La inhibición de la $\mathrm{H}+\mathrm{K}+\mathrm{ATPasa}$ resulta en la reducción de secreción ácida gástrica (Earnest, 1990) (22); los hallazgos indican que el extracto ha mejorado la presencia de lesiones gástricas inducidas por etanol, indicando su importancia que estaría inhibiendo mecanismos involucrados con la estimulación de la $\mathrm{H}+\mathrm{K}+\mathrm{ATPasa}$ y por tanto reduciendo la secreción ácido gástrica, lo que ha conllevado a menos lesión (tabla 4, 5; figuras $1,2)$. Se hace necesario profundizar en esta investigación para arribar a mejores conclusiones y explicaciones de los mecanismos de acción del extracto.

Luego de extraer los estómagos de los animales de experimentación se observa que a diferentes dosis administradas hay una gastroprotección directamente proporcional a la concentración de extracto. En las dosis de $25 \mathrm{mg}$ de extracto hidroalcohólico/Kg de peso se observa muy poca 
gastroprotección, las zonas congestivas son parecidas a la del control úlcera. La dosis de $250 \mathrm{mg}$ de extracto hidroalcohólico/Kg de peso muestra mejorías respecto a la dosis anterior, son zonas en donde existen menores zonas congestivas e hiperémicas. Pero, en las dosis de $1000 \mathrm{mg}$ de extracto $/ \mathrm{Kg}$ de peso se aprecia una gastroprotección que sobrepasa el $90 \%$, en algunos casos se podría hablar hasta de un $100 \%$ (tabla 4 ). Como se observa en la tabla 5, las características histopatológicas luego del tratamiento a tres dosis sobre la mucosa gástrica, muestran las lesiones gástricas, a dosis altas se ven fragmentos de mucosa suelta pero en áreas muy pequeñas siendo el resto normal, luego el extracto hidroalcohólico de Satureja sericea cumple la actividad como gastroprotector, por lo tanto, se confirma las propiedades carminativas $\mathrm{y}$ antiinflamatorias que se presentan generalmente en especies del género Satureja (15-17).

En conclusión, el extracto hidroalcohólico de $S a$ tureja sericea contiene una mayor proporción de compuestos fenólicos, principalmente flavonoides, cuyas fracciones obtenidas son: M1. $3^{\prime}, 4^{\prime}, 5$, 7- tetrahidroxi-6,8- di metoxi flavona; M2. 7- hidroxi- 3',5,6- tri metoxi flavona; M3. 7-hidroxi$3^{\prime}, 5,6,8$ - tetra metoxi flavona y M4. $3^{\prime}, 4^{\prime}, 5,7-$ tetrahidroxi- 8 - metoxi flavona; además se ha comprobado la actividad gastroprotectora de los flavonoides presentes en el extracto hidroalcohólico de Satureja sericea. 


\section{REFERENCIAS BIBLIOGRÁFICAS}

1. Maureen H, García L, Rojo D, Olivares D. 2003. Almendro de la india potencial biológico valioso. Centro de investigaciones biomédicas. Instituto de Ciencias Básicas y Preclínicas "Victoria de Girón". Revista Cubana Investigaciones Biomédicas. 22(1):41-7.

2. Cotram, Kusmart y Robbins. 1992. Patología Estructural y Funcional. New York: Mac Graw Hill Interamericana. p. 816, 823,824.

3. Sistema Virtual de Bibliotecas, UNMSM. Loja B. 2002. Contribución al estudio florístico de la provincia de Concepción, (Junín): Dicotiledóneas [Revisado 23 mar 2008] http://www.cybertesis. edu.pe/sdx/sisbib/notice. $x$ sp?id=sisbib. 2002 . loja_Satureja sericea.

4. Basten J. 1981. Las Plantas Medicinales de Ios Kallawayas, La Paz: 1st ed. Proyecto Concern Bolivia. p. 145.

5. Gupta M P. 1995. 270 Plantas Medicinales Iberoamericanas. In: M.P. Gupta (Ed.), Bogotá: Convenio Andres Bello, Colombia. p. 112, 126, 162.

6. Lock de Ugaz O. 1994. Investigación Fitoquímica. Métodos en el estudio de productos naturales. Lima: Fondo Editorial PUCP. 2a Edición. p.5.

7. Villar del Fresno M. 1999. Farmacognosia General. Madrid: Editorial Síntesis.

8. Kuklinski C. 2000. Farmacognosia. Estudio de las Drogas y Sustancias Medicamentosas. Barcelona: Ediciones Omega.

9. Bruneton J. 2001. Plantas Medicinales. Fitoquímica y Farmacognosia. $2^{a}$ Edición, Zaragoza: Editorial Acribia S A.

10. Brako L, Zaruchi J L. 1993. Catalogue of Flowering Plants. Angiosperms and Gymnosperms of Perú: Kansas City: Monographs in Sistematic Botany from the Missouri Botanical Garden.

11. Domínguez XA. 2000. Métodos de Investigación Fitoquímica. México DF: Editorial Limusa SA.

12. Mabry TJ, Markham KR, Thomas MB. 1970. The Systematic Identification of Flavonoids. New York: Edit. Springer-Verlag. New York-Heidelberg-Berlin.

13. Zhang SR, Cui GJ, Xu RM, Hang C, Guo JY. 1995. Antiulcer effect of polycyclicamine com- pound HHO1 on experimental gastric ulcer in rats. Yao Xue Xue Bao. 30: 103-106.

14. Liu CF, Lin CC, Lin SC. 2002. Protection by Tetramethylpyrezine in Acute Absolute EtanolInduced Gastric Lesions. J Biomed Sci. 9: 395400.

15. Casa Pía. Satureja Montana [Revisado 15 may 2008] Disponible en la World Wide Web: http://www.casapia.com/dietetica-herbolario/ las-plantas-medicinales/ajedrea-satureja-montana-l.html

16. Info Jardín. Satureja Hortensis [Revisado 15 may 2008] Disponible en la World Wide Web: http://fichas.infojardin.com/condimentos/satureja-hortensis-ajedrea-blanca-ajedrea-comunjardin.htm

17. Fresquet JL, Blanquer $G$, Galindo $M$, Gallego F, García de la Cuadra R, López JA, Sanjosé A. Universidad de Valencia. Inventario de las plantas de medicinales de uso popular en la ciudad de Valencia [Revisado 16 may 2008] mayo, 2001. Disponible en la Word Wide Web: http:/ / www. uv.es/medciensoc/num2/inventario.PDF.

18. Bandhopadhyay $U$, Biswas $K$, Chatterjee $R$, Kumar Ganguly ICC, Bhattacharya K, Banerjee R. 2002. Gastroprotective effect of Neem (Azadiracta indica) bark extract: possible involvement of $\mathrm{H}+\mathrm{K}+\mathrm{ATPase}$ inhibition and scavenging of hydroxyl radical. Life Science . 71: 2845-2865.

19. Nagaya H, Satoh H, Maki Y. 1990. Possible mechanism for the inhibition of acid formation by the proton pump inhibitor AG-1749 in isolated canine parietal cells. Journal of Pharmacology and Experimental Therapeutics. 252: 1289-1295. 20. Brunton LL. 1966. Pharmacological treatment of heart failure. In: Hardman, J.G., Gilman, A.G., Limbird, L.E. (Eds.), Goodman and Gilmans: The Pharmacologic Basis of Therapeutics, New York: 9th ed. McGraw-Hill. p. 901-915.

21. David Perlin S. 1998. Ion pumps as targets for therapeutic intervention: Old and new paradigms. Electronic Journal of Biotechnology. 1: 55-64.

22. Earnest DL. 1990. NSAID-induced gastric injury: Its pathogenesis and management. Seminars in Arthritis and Rheumatism. 19: 6-10. 